\title{
A reduction of the NF consistency problem
}

\author{
Athanassios Tzouvaras \\ Dept. of Mathematics, Univ. of Thessaloniki, \\ 54124 Thessaloniki, Greece. \\ e-mail:tzouvara@math.auth.gr
}

\begin{abstract}
We give a necessary and sufficient condition in order that a typeshifting automorphism be constructed on a model of the Theory of Simple Types (TST) by forcing. Namely it is proved that, if for every $n \geq 1$ there is a model of TST in the ground model $M$ of ZFC that contains an $n$-extendible coherent pair, then there is a generic extension $M[G]$ of $M$ that contains a model of TST with a type-shifting automorphism, and hence $M[G]$ contains a model of NF. The converse holds trivially. It is also proved that there exist models of TST containing 1-extendible coherent pairs.
\end{abstract}

Keywords. New Foundations, type-shifting automorphism, forcing, coherent pair, $n$-extendible coherent pair.

\section{Introduction and preliminaries}

The major open problem of the theory NF (New Foundations) is its consistency (relative to that of ZFC). Important reductions of the problem were obtained by E. Specker (who reduced it to the consistency of TST+(Amb)), and by V.N. Grishin (who reduced NF to its fragment $\mathrm{NF}_{4}$ ). In this paper we use both of the aforementioned reductions and give a new one using the language of forcing. Actually the reduction emerged out from an attempt to construct a type-shifting automorphism on a model of $\mathrm{TST}_{4}$ (the fragment of the theory of types TST up to fourth level) by forcing. The main implication reads as follows: "If for each $n \geq 1$ there is a model of TST in the 
ground model $M$ of ZFC that contains an $n$-extendible coherent pair, then there is a generic extension $M[G]$ of $M$ that contains a model of TST with a type-shifting automorphism, and hence $M[G]$ contains a model of NF". The converse holds trivially: If $M$ contains a model of $\mathrm{NF}$, then it contains also a model of TST having $n$-extendible coherent pairs, for every $n \geq 1$. Coherent pairs are, roughly, finite approximations of a type-shifting automorphism of a model of $\mathrm{TST}_{4}$.

Section 1 contains preliminary material. Most of it is well-known, but some facts, though elementary, seem to have been fixed here for the first time. For example the definition 1.1 of level collapse of a model, and lemmas 1.2 and 1.6, which essentially allow one to restrict oneself to standard transitive models of TST. Also lemma 1.9 is just an adaptation of a result of Grishin.

In section 2 we define the notion of $n$-extendible coherent pair and, by the help of a certain extension of the theory TST, we prove the main theorem 2.8 (and its equivalent version theorem 2.9).

In section 3 we prove that there are models of TST containing 1-extendible (i.e., just extendible) coherent pairs (theorem 3.6).

Section 4, finally, contains some comments on 2-extendible coherent pairs.

\subsection{The theory of types and its models}

Our metatheory will be ZFC. We often refer to it either as "external world" or as "ground model". $\in$ is the membership relation of the ground model. $\mathbb{N}$ is the set of natural numbers of the ground model.

The language $L_{\mathrm{TST}}$ of the Theory of Simple Types (TST) consists of the binary predicate symbol $\varepsilon$ and countably many sorts (or types) $S_{i}(x)$, $i \in \mathbb{N}$. Usually we introduce typed variables $x^{i}$, $y^{j}$, etc., $i, j \in \mathbb{N}$, where the superscript indicates the type, and $x^{i}$ stands for $S_{i}(x)$. Formulas of $L_{\mathrm{TST}}$ are built from atomic formulas of the form $x^{i} \varepsilon x^{i+1}$ and $x^{i}=y^{i}$, in the ordinary way. The axioms of TST are the following schemes of comprehension and extensionality:

(Co) $\left(\exists x^{i+1}\right)\left(\forall y^{i}\right)\left(y^{i} \varepsilon x^{i+1} \Leftrightarrow \phi\left(y^{i}\right)\right)$, for every $\phi\left(y^{i}\right) \in L_{\text {TST }}$ possibly with extra free variables.

(Ex) $\quad\left(\forall x^{i}\right)\left(x^{i} \varepsilon y^{i+1} \Leftrightarrow x^{i} \varepsilon z^{i+1}\right) \Rightarrow y^{i+1}=z^{i+1}$.

A model of TST is a sequence $\mathcal{A}=\left(A, A_{0}, A_{1}, \ldots, R\right)$, such that $A=$ $\bigcup_{i \in \mathbb{N}} A_{i}$, and each $A_{i}$ interprets the variables of type $i$. $R$ is a binary relation 
on $A$, i.e., $R \subseteq A^{2}$, that interprets $\varepsilon$. Since $A=\bigcup_{i \in \mathbb{N}} A_{i}$, we may just write $\mathcal{A}=\left(A_{0}, A_{1}, \ldots, R\right)$ instead of $\mathcal{A}=\left(A, A_{0}, A_{1}, \ldots, R\right)$. Note that the axioms above say nothing as to whether the levels $A_{i}$ of a model are disjoint or not, or whether there are $x, y$ belonging to the same level such that $x R y$. So $R$ may well be non-well-founded.

For every $i \geq 0$ and $x \in A_{i+1}$ let

$$
x_{R}=\left\{y \in A_{i}: y R x\right\} .
$$

$\mathcal{A}$ is said to be standard if $R$ is $\in$ (restricted to the sets of the model). Equivalently $\mathcal{A}$ is standard if for every $x \in A_{i+1}$,

$$
x_{R}=x \cap A_{i} .
$$

A standard $\mathcal{A}$ is transitive if for every $i, x \in A_{i+1} \Rightarrow x \subseteq A_{i}$, i.e., if for every $x \in A_{i+1}$,

$$
x_{R}=x .
$$

In such a case for all $i, A_{i+1} \subseteq \mathcal{P}\left(A_{i}\right)$.

Standard transitive (henceforth s.t.) models of TST are the most natural and intuitively graspable ones. E.g. for every $X \neq \emptyset$, the sequence $\left(X, \mathcal{P}(X), \mathcal{P}^{2}(X), \ldots, \in\right)$ is a s.t. model of TST. Such a model is called full and is denoted by $\langle\langle X\rangle\rangle$. If $X$ is infinite $\langle\langle X\rangle\rangle$ is uncountable. To find a countable model we can take a countable elementary submodel of $\langle\langle X\rangle\rangle$. Such a model is standard but not transitive.

Definition 1.1 Given a model $\mathcal{A}=\left(A_{0}, A_{1}, \ldots, R\right)$ of TST, define the mapping $\sigma: \bigcup_{i} A_{i} \rightarrow V$ by induction on $i$ as follows:

$$
\begin{aligned}
& \sigma(a)=a \text { for all } a \in A_{0}, \\
& \sigma(x)=\left\{\sigma(y): y \in A_{i} \& y R x\right\}=\left\{\sigma(y): y \in x_{R}\right\}, \text { for } x \in A_{i+1} .
\end{aligned}
$$

Let $B_{i}=\sigma^{\prime \prime} A_{i}{ }^{1}$ and $\mathcal{B}=\left\{B_{0}, B_{1}, \ldots, \in\right)$. $\mathcal{B}$ is said to be the level collapse of $\mathcal{A}$ and is denoted by $l c(\mathcal{A})$.

Obviously $l c(\mathcal{A})$ is a standard and transitive structure, defined independently of whether $R$ is well-founded or not ${ }^{2}$. In particular, if $\mathcal{A}$ is standard,

\footnotetext{
${ }^{1}$ Given a mapping $h: X \rightarrow Y, h^{\prime \prime}$ denotes the induced mapping from $\mathcal{P}(X)$ to $\mathcal{P}(Y)$, where $h^{\prime \prime} x=\{h(y): y \in x\}$ (T. Forster [1] uses the notation $j^{\text {' }} h$ for $h^{\prime \prime}$ ). If $h$ is $1-1$ or onto, then so is $h^{\prime \prime}$.

"If the relation $R$ of $\mathcal{A}$ is well-founded, then the usual "Mostowski collapse" $\pi$ can also be defined on $A$ by induction on the $R$-rank of the elements of $A$. Namely $\pi(x)=$ $\{\pi(y): y R x\}$. Then for every $i$ and every $x \in A_{i}$ and $y \in A_{i+1}, x R y \Longleftrightarrow \pi(x) \in \pi(y)$. However $\pi$ may distort heavily $\mathcal{A}$. For example if all elements of $A_{0}$ are $R$-minimal, then $\pi^{\prime \prime} A_{0}=\{\emptyset\}$. So this kind of collapse has no practical use.
} 
then for $x \in A_{i+1}, \sigma(x)=\left\{\sigma(y): y \in x \cap A_{i}\right\}$. Since $\mathcal{A} \models \mathrm{Ex}$, it is easy to see that for every $i, \sigma \mid A_{i}$ is 1-1. But $\sigma$ need not be $1-1$ on the entire $\bigcup_{i} A_{i}$. For example, for each $i$ there is an object $x \in A_{i}$ such that $x_{R}=\emptyset$. Then for every such $x, \sigma(x)=\emptyset$.

Lemma 1.2 Let $\mathcal{A} \models$ TST. For every formula $\phi\left(x_{1}^{i_{1}}, \ldots, x_{n}^{i_{n}}\right)$ of $L_{\mathrm{TST}}$, with free variables among $x_{1}^{i_{1}}, \ldots, x_{n}^{i_{n}}$, and every sequence of objects $a_{1}, \ldots, a_{n}$ such that $a_{k} \in A_{i_{k}}$,

$$
\mathcal{A} \models \phi\left(a_{1}, \ldots, a_{n}\right) \Longleftrightarrow l c(\mathcal{A}) \models \phi\left(\sigma\left(a_{1}\right), \ldots, \sigma\left(a_{n}\right)\right) .
$$

Proof. By induction on the length of $\phi$. Consider the atomic formula $x^{i} \varepsilon y^{i+1}$. Then for every $a \in A_{i}$ and every $b \in A_{i+1}$ we have, by the definition of $\sigma$,

$$
\mathcal{A}=a \varepsilon b \Longleftrightarrow a R b \Longleftrightarrow \sigma(a) \in \sigma(b) \Longleftrightarrow l c(\mathcal{A}) \models \sigma(a) \varepsilon \sigma(b) .
$$

Similarly for the atomic formula $x^{i}=y^{i}$, and any $a, b \in A_{i}$ we have

$$
\mathcal{A} \models a=b \Longleftrightarrow a=b \Longleftrightarrow l c(\mathcal{A}) \models \sigma(a)=\sigma(b) .
$$

The other steps of the induction are routine.

It follows from lemma 1.2 that for every model $\mathcal{A}$ of TST, not only is $l c(\mathcal{A})$ a s.t. model of TST, but also $\mathcal{A}$ and $l c(\mathcal{A})$ are "almost isomorphic". In view of this fact, and also of the lemma 1.6 below, talking about models of TST can be practically restricted to talking about s.t. models only. So henceforth, unless otherwise stated, a model of TST will be a s.t. one. Moreover, in cases where we write formulas of $L_{\mathrm{TST}}$ semi-formally, we conflate $\varepsilon$ with $\in$.

Let $\mathcal{A}=\left(A_{0}, A_{1}, \ldots\right)$ be a model of TST. A set $X \subseteq A_{i}$ is said to be definable in $\mathcal{A}$ if $X=\left\{x \in A_{i}: \mathcal{A}=\phi(x)\right\}$ for some formula $\phi\left(x^{i}\right)$, possibly with parameters. In view of the axiom (Co), $X$ is definable in $\mathcal{A}$ iff $X$ belongs to (some level of) $\mathcal{A}$. For example if $f: A_{i} \rightarrow A_{i}$ is a definable function, then $f \in A_{i+3}$. Sets containing objects from various levels of $\mathcal{A}$ cannot belong to $\mathcal{A}$ (being non-stratified objects). Nevertheless we can extend to them also the property of definability, by considering certain stratified copies of them. Such copies are constructed using the "shifting mapping" $\imath(x)=\{x\}$ and its iterations. To be specific, a mapping $f: A_{i} \rightarrow A_{i+1}$ is said to be definable if the mapping $g: \imath^{\prime \prime} A_{i} \rightarrow A_{i+1}$ such that $g(\imath(x))=f(x)$ (i.e., $g \circ \imath=f$ ) is 
definable. E.g. $\imath$ itself is definable, since the identity $i d$ for which $i d \circ \imath=\imath$ is definable.

In every structure $\mathcal{A}=\left(A_{0}, A_{1}, \ldots\right)$, each $A_{i+1}$ plays, roughly, the role of the powerset of $A_{i}$. So although bijections $f: A_{i} \rightarrow A_{i+1}$ always exist for countable $\mathcal{A}$, none of them can be definable in $\mathcal{A}$, because otherwise Cantor's diagonal argument reappears.

Lemma 1.3 Let $\mathcal{A}=\left(A_{0}, A_{1}, \ldots\right)$ be a model of TST. For every $i$, there is no definable bijection $f: A_{i} \rightarrow A_{i+1}$.

Proof. Suppose $f: A_{i} \rightarrow A_{i+1}$ is a definable bijection. Then there is a definable bijection $g: \imath^{\prime \prime} A_{i} \rightarrow A_{i+1}$. By (Co) there is $y \in A_{i+1}$ such that $y=\left\{x \in A_{i}: x \notin g(\imath(x))\right\}$. Hence there is $c \in A_{i}$ such that $g(\imath(c))=y$. Then for every $x \in A_{i}$,

$$
x \in y \Longleftrightarrow x \in g(\imath(c)) \Longleftrightarrow x \notin g(\imath(x)) .
$$

For $x=c$ we have $c \in g(\imath(c)) \Longleftrightarrow c \notin g(\imath(c))$, a contradiction.

The discussion in subsequent sections involves the property of "finiteness" of sets of a model $\mathcal{A}$. Every model of TST has an internal notion of finiteness, expressed by a formula Fin $\left(x^{i+1}\right)$ of $L_{\mathrm{TST}}$. This is defined in terms of the operation $S$ defined on each $A_{i+2}$ as follows (we drop the type superscripts for readability and write $\in$ instead of $\varepsilon$ ):

$$
S(z)=\left\{y:\left(\exists y_{1} \in z\right)\left(\exists x \notin y_{1}\right)\left(y=y_{1} \cup\{x\}\right)\right\} .
$$

Intuitively, $S$ sends the class of all sets (of some level) with $n$ elements, to the class of sets (of the same level) with $n+1$ elements. For example, if $z=\{\emptyset\}$, then $S(z)$ is the class of singletons, and so on. Now a set is finite if it belongs to the intersection of all sets which contain $\emptyset$ and whenever they include $y$, they include also $S(y)$. Formally (dropping superscripts again)

$$
\operatorname{Fin}(x) \Longleftrightarrow \forall z[\emptyset \in z \wedge(\forall y)(y \subseteq z \Rightarrow S(y) \subseteq z) \Rightarrow x \in z] .
$$

In contrast to Fin $(x)$, we have also the external notion of finiteness (i.e., with respect to the ground model $M$ ), which we express by writing simply " $x$ is finite". For every model $\mathcal{A}$ of TST and every $x \in A_{i+1}$, one can easily see by induction on $|x|$ that 


$$
x \text { is finite } \Rightarrow \mathcal{A}=\operatorname{Fin}(x) .
$$

The converse of (1) is false in general ${ }^{3}$. However there are models $\mathcal{A}$ for which the converse of (1) is also true, e.g. the full models $\langle\langle X\rangle\rangle$. Such models, for which finiteness is absolute, are considered in section 3, where they are called "regular".

\subsection{The system NF}

The language $L_{\mathrm{NF}}$ of $\mathrm{NF}$ consists of the predicate $\varepsilon$ and untyped variables $x, y, \ldots$. A formula of $L_{\mathrm{NF}}$ is called stratified if it results from a formula of $L_{\mathrm{TST}}$ if we erase all type superscripts from its variables. The axioms of NF are stratified comprehension and extensionality:

(StCo) $\quad(\exists x)(\forall y)(y \varepsilon x \Leftrightarrow \phi(y))$, for every stratified $\phi(y) \in L_{\mathrm{NF}}$, possibly with extra free variables.

$(\mathrm{Ex})(\forall x)(x \varepsilon y \Leftrightarrow x \varepsilon z) \Rightarrow y=z$.

Given a model $\mathcal{A}=\left(A_{0}, A_{1}, \ldots\right)$ of TST, let $\mathcal{A}^{+}=\left(A_{1}, A_{2}, \ldots\right) . \mathcal{A}^{+}$is still a model of TST.

Given a formula $\phi \in L_{\text {TST }}$ let $\phi^{+}$denote the formula resulting from $\phi$ if we raise the type of each variable of $\phi$ by one. If TST $\vdash \phi$ then TST $\vdash \phi^{+}$. But the converse is false. The axiom scheme:

(Amb) $\phi \Leftrightarrow \phi^{+}, \phi \in L_{\mathrm{TST}}$,

is called Typical Ambiguity.

Definition 1.4 Let $\mathcal{A}=\left(A_{0}, A_{1}, \ldots, R\right)$ be a model of TST (not necessarily s.t.) A type-shifting automorphism (or just an automorphism ) of $\mathcal{A}$ is a sequence of mappings $f=\left(f_{0}, f_{1}, \ldots\right)$ such that:

(a) each $f_{i}$ is $1-1$,

(b) $\operatorname{dom}\left(f_{i}\right)=A_{i}$,

(c) $\operatorname{rng}\left(f_{i}\right)=A_{i+1}$,

(d) $x R y \Longleftrightarrow f_{i}(x) R f_{i+1}(y)$ for every $x \in A_{i}$ and $y \in A_{i+1}$.

We often denote this automorphism by $A_{0} \stackrel{f_{0}}{\longrightarrow} A_{1} \stackrel{f_{1}}{\longrightarrow} A_{2} \stackrel{f_{2}}{\longrightarrow} \cdots$

\footnotetext{
${ }^{3}$ See e.g. the model that Grishin constructs in [2], in which $A_{0}$ is externally infinite and yet $\mathcal{A} \models$ Fin $(x)$ holds for every $x \in A_{i+1}$.
} 
The following fundamental result of E. Specker relates TST, typical ambiguity, and NF.

\section{Theorem 1.5 (Specker [4])}

The following are equivalent:

(i) NF is consistent.

(ii) There is a model $\mathcal{A}$ of TST possessing an automorphism $f: \mathcal{A} \rightarrow \mathcal{A}^{+}$.

(iii) There is a model $\mathcal{A}$ of TST such that $\mathcal{A} \equiv \mathcal{A}^{+}$.

(iv) TST + Amb is consistent.

The difficult and most important implication is (iv) $\Rightarrow$ (ii). For a proof see [4], or [1], p. 58 .

Lemma 1.6 For every model $\mathcal{A}$ of TST, there exists an automorphism on $\mathcal{A}$ iff there exists an automorphism on $\operatorname{lc}(\mathcal{A})$.

Proof. Let $\mathcal{A}=\left(A_{0}, A_{1}, \ldots, R\right)$ be a model of TST and let

$$
A_{0} \stackrel{f_{0}}{\longrightarrow} A_{1} \stackrel{f_{1}}{\longrightarrow} A_{2} \stackrel{f_{2}}{\longrightarrow} \cdots
$$

be a an automorphism. Let $l c(\mathcal{A})=\left(B_{0}, B_{1}, \ldots\right)$ and let $\sigma: \mathcal{A} \rightarrow l c(\mathcal{A})$ be the level collapsing mapping. Put $g_{i}=\sigma \circ f_{i} \circ \sigma^{-1}$, for every $i$. Since $\sigma$ is 1-1 on each level $A_{i}, g_{i}$ is well-defined. And clearly $g_{i}: B_{i} \rightarrow B_{i+1}$ is a bijection. Moreover, in view of lemma 1.2, for every $x \in B_{i}$ and every $y \in B_{i+1}$,

$$
\begin{gathered}
x \in y \Longleftrightarrow \sigma^{-1}(x) R \sigma^{-1}(y) \Longleftrightarrow f_{i} \sigma^{-1}(x) R f_{i+1} \sigma^{-1}(y) \Longleftrightarrow \\
\sigma f_{i} \sigma^{-1}(x) \in \sigma f_{i+1} \sigma^{-1}(y) \Longleftrightarrow g_{i}(x) \in g_{i+1}(y) .
\end{gathered}
$$

Therefore

$$
B_{0} \stackrel{g_{0}}{\longrightarrow} B_{1} \stackrel{g_{1}}{\longrightarrow} B_{2} \stackrel{g_{2}}{\longrightarrow} \cdots
$$

is an automorphism. The converse is similar. Given the automorphism $\left(g_{0}, g_{1}, \ldots\right)$ of $l c(\mathcal{A})$, it suffices to define $f_{i}=\sigma^{-1} \circ g_{i} \circ \sigma$.

In view of lemma 1.6, theorem 1.5 remains true if we replace the word "model" by "s.t. model" everywhere.

If $\mathcal{A}$ is s.t. and $f: \mathcal{A} \rightarrow \mathcal{A}^{+}$is an a automorphism, then condition (d) of definition 1.4 becomes

$$
x \in y \Longleftrightarrow f_{i}(x) \in f_{i+1}(y)
$$


for every $x \in A_{i}$ and $y \in A_{i+1}$. Since $f_{i}$ are onto, this is equivalent to the property that for every $x \in A_{i+1}$,

$$
f_{i+1}(x)=f_{i}^{\prime \prime} x
$$

i.e., that $f_{i+1}=f_{i}^{\prime \prime}$.

\subsection{Fragments of NF}

For $n>0$, a formula $\phi$ of $L_{\mathrm{TST}}$ is an $n$-formula, if every variable of $\phi$ is of type $<n$. Let $\mathrm{TST}_{n}$ be the subtheory of TST whose axioms are those of TST restricted to $n$-formulas. A model of $\operatorname{TST}_{n}$ is an $n$-sequence $\mathcal{A}=$ $\left(A_{0}, A_{1}, \ldots, A_{n-1}\right)$.

Similarly, a formula $\phi$ of $L_{\mathrm{NF}}$ is $n$-stratified if it results from an $n$-formula of TST by erasing the types from the variables. $\mathrm{NF}_{n}$ is the subtheory of $\mathrm{NF}$ in which the scheme of Stratified Comprehension is restricted to $n$-stratified formulas.

An easy consequence of theorem 1.5 is the following.

Corollary 1.7 For every $n, \mathrm{NF}_{n}$ is consistent iff there is a model $\left(A_{0}, A_{1}, \ldots, A_{n-1}\right)$ of $\mathrm{TST}_{n}$ possessing an automorphism $f:\left(A_{0}, A_{1} \ldots, A_{n-2}\right) \rightarrow\left(A_{1}, A_{2} \ldots, A_{n-1}\right)$.

Grishin [2] has shown that $\mathrm{NF}_{3}$ is consistent, and that $\mathrm{NF}$ is equivalent to $\mathrm{NF}_{4}$.

Theorem 1.8 (Grishin) (a) $\mathrm{NF}_{3}$ is consistent.

(b) $\mathrm{NF}=\mathrm{NF}_{4}=\mathrm{NF}_{3}+\exists z z=\{\{\{x\}, y\}: x \varepsilon y\}$.

The following result is an adaptation of an idea of Grishin's (used in [2] to prove 1.8 (a)), to the fragment $\mathrm{NF}_{4}$.

Lemma 1.9 $\mathrm{NF}$ is consistent iff there is a model $\mathcal{A}=\left(A_{0}, A_{1}, \ldots\right)$ of TST and a pair of bijections $A_{1} \stackrel{f_{1}}{\longrightarrow} A_{2} \stackrel{f_{2}}{\longrightarrow} A_{3}$ such that for all $x, x_{1}, x_{2} \in A_{1}$ and all $y \in A_{2}$

$$
\begin{aligned}
x \in y & \Longleftrightarrow f_{1}(x) \in f_{2}(y), \\
x_{1} \subseteq x_{2} & \Longleftrightarrow f_{1}\left(x_{1}\right) \subseteq f_{1}\left(x_{2}\right) .
\end{aligned}
$$


Proof. If NF is consistent then, by 1.5 , there is a model $\mathcal{A}=\left(A_{0}, A_{1}, \ldots\right)$ of TST with an automorphism $f=\left(f_{0}, f_{1}, f_{2}, \ldots\right)$. Then clearly the pair $\left(f_{1}, f_{2}\right)$ satisfies $(3)$ and $(4)$.

Conversely, suppose there is a model $\mathcal{A}=\left(A_{0}, A_{1}, \ldots\right)$ of TST and a pair of bijections $A_{1} \stackrel{f_{1}}{\longrightarrow} A_{2} \stackrel{f_{2}}{\longrightarrow} A_{3}$ satisfying (3) and (4). Then $\left(A_{0}, A_{1}, A_{2}, A_{3}\right)$ is a model of $\mathrm{TST}_{4}$. As Grishin observed in [2] (for the case of $\mathrm{NF}_{3}$ and $\mathrm{TST}_{3}$, respectively), if we define $f_{0}: A_{0} \rightarrow A_{1}$ by setting

$$
f_{0}(a)=x \Longleftrightarrow f_{1}(\{a\})=\{x\}
$$

then $f_{0}$ is a bijection because, by (4), $f_{1}$ sends atoms to atoms. Moreover $\left(f_{0}, f_{1}, f_{0}\right)$ is an automorphism from $\left(A_{0}, A_{1}, A_{2}\right)$ onto $\left(A_{1}, A_{2}, A_{3}\right)$. Indeed, by the definition of $f_{0}$ we have that for every $a, f_{1}(\{a\})=\left\{f_{0}(a)\right\}$. So for every $a \in A_{0}$ and $x \in A_{1}$

$$
f_{0}(a) \in f_{1}(x) \Longleftrightarrow\left\{f_{0}(a)\right\} \subseteq f_{1}(x) \Longleftrightarrow f_{1}(\{a\}) \subseteq f_{1}(x) .
$$

Now by (4),

$$
f_{1}(\{a\}) \subseteq f_{1}(x) \Longleftrightarrow\{a\} \subseteq x \Longleftrightarrow a \in x .
$$

Therefore combining the above equivalences we get

$$
a \in x \Longleftrightarrow f_{0}(a) \in f_{1}(x)
$$

The last equivalence and (3) show that $\left(f_{0}, f_{1}, f_{2}\right)$ is an automorphism $\left(A_{0}, A_{1}, A_{2},\right) \rightarrow\left(A_{1}, A_{2}, A_{3}\right)$. By 1.7, this implies that there is a model of $\mathrm{NF}_{4}$, and hence, by 1.8 (b), there is a model of NF.

In the sequel, when a pair of bijections $\left(f_{1}, f_{2}\right)$ satisfies conditions $(3)$ and (4) above, we shall say that $f_{1}$ and $f_{2}$ are $\in$ - and $\subseteq$-isomorphisms.

\section{Attacking the NF consistency problem with forcing}

Let $\mathcal{A}=\left(A_{0}, A_{1}, A_{2}, A_{3}\right)$ be a model of $\mathrm{TST}_{4}$ (or more generally a model $\mathcal{A}=\left(A_{0}, A_{1}, A_{2}, \ldots\right)$ of TST). By lemma 1.9 , in order to turn $\mathcal{A}$ into a model of $\mathrm{NF}_{4}$, and hence of $\mathrm{NF}$, it suffices to construct a pair of bijections 
$A_{1} \stackrel{f_{1}}{\longrightarrow} A_{2} \stackrel{f_{2}}{\longrightarrow} A_{3}$ satisfying conditions (3) and (4). Now it is natural to attempt to construct $\left(f_{1}, f_{2}\right)$ by forcing, i.e., via its finite (and not only finite) parts.

The idea is the usual one: If we start with a model $M$ of ZFC containing $\mathcal{A}=\left(A_{0}, A_{1}, A_{2}, A_{3}\right)$, and we are able to find an appropriate set of forcing conditions $(P, \leq)$ consisted of parts of the pair $\left(f_{1}, f_{2}\right)$, then $\left(f_{1}, f_{2}\right)$ will emerge as a generic subset of $P$, and hence a model of NF will appear in the extension $M[G]$ of $M$.

However, the preceding idea comprises two main steps: (a) To define an appropriate set of forcing conditions, and (b) to show that the generic set does the job it was designed to, i.e., provides an automorphism for the underlying model.

Concerning step (a), the forcing conditions are going to be not all, but some (if any) of the finite approximations of the sought pair $\left(f_{1}, f_{2}\right)$. So we shall define first certain pairs of functions $p=\left(p_{1}, p_{2}\right)$, that we shall call "coherent pairs". We do not yet call $\left(p_{1}, p_{2}\right)$ "forcing conditions" because they lack in general the key property that forcing conditions ought to have, namely extendibility. So in section 2.1 we introduce coherent pairs. In fact in order to speak about them formally a certain extension of the theory TST and its language is needed.

Step (b) concerns the crucial property of extendibility of coherent pairs. We deal with this in section 2.2. A pair $p$ is extendible if for every element $t$ of the model, there is a pair $q$ that extends $p$ and captures $t$. Stronger notions of $n$-extendibility, for $n \geq 1$, and $\omega$-extendibility are introduced. A pair $p$ is $(n+1)$-extendible if for every element $t$, there is an $n$-extendible pair $q$ that extends $p$ and captures $t . p$ is $\omega$-extendible, if it is $n$-extendible for every $n \geq 1$. The reduction of NF consistency problem stated in the title, consists in replacing it with the problem of whether, for every $n \geq 1$, there is a model $\mathcal{A}$ of TST containing an $n$-extendible coherent pair. If this is the case, then there is a model $\mathcal{B}$ of TST containing $\omega$-extendible coherent pairs. Then the set $P_{\omega}$ of $\omega$-extendible coherent pairs can be used as the set of forcing conditions, and any generic $G \subseteq P_{\omega}$ provides a triple $\left(f_{0}, f_{1}, f_{2}\right)$ of isomorphisms for the structure $\left(B_{0}, B_{1}, B_{2}, B_{3}\right)$. (Actually $G$ provides the pair $\left(f_{1}, f_{2}\right) . f_{0}$ is trivially defined through $f_{1}$.) So a model of $\mathrm{NF}_{4}$ (and hence of NF) exists in the generic extension $M[G]$ of the ground model $M$.

To summarize: If for every $n \in \mathbb{N}, n$-extendible coherent pairs exist in a model $M$ of ZFC, then there is a model of NF in a generic extension $M[G]$ of 
$M$. The converse is also true rather trivially: If $M$ contains a model of NF, then it contains a model $\mathcal{A}$ of TST in which there are $n$-extendible coherent pairs, for every $n \geq 1$. This is briefly the content of the main theorem 2.8 .

\subsection{Coherent pairs}

Throughout $M$ will be a fixed countable s.t. model of ZFC and $\mathcal{A}=$ $\left(A_{0}, A_{1}, \ldots\right) \in M$ will be a countable (in the sense of $\left.M\right)$ s.t. model of TST. In view of lemma 1.9, in order for $\mathcal{A}$ to be turned into a model of NF, it suffices that a pair of bijections $A_{1} \stackrel{f_{1}}{\longrightarrow} A_{2} \stackrel{f_{2}}{\longrightarrow} A_{3}$ be constructed satisfying conditions (3) and (4). Then the parts ${ }^{4}$ of $\left(f_{1}, f_{2}\right)$ should also satisfy conditions (3) and (4). The parts of $\left(f_{1}, f_{2}\right)$ will be called "coherent pairs" and will be denoted $p=\left(p_{1}, p_{2}\right)$. Thus, a coherent pair of $\left.\mathcal{A}\right)$ is a pair of functions $p=\left(p_{1}, p_{2}\right)$, such that $\operatorname{dom}\left(p_{1}\right) \subseteq A_{1}, \operatorname{dom}\left(p_{2}\right)=\operatorname{rng}\left(p_{2}\right) \subseteq A_{2}$, $r n g\left(p_{2}\right) \subseteq A_{3}$, and for all $x \in \operatorname{dom}\left(p_{1}\right)$ and $y \in \operatorname{dom}\left(p_{2}\right)$

$$
x \in y \Longleftrightarrow p_{1}(x) \in p_{2}(y),
$$

and for all $x_{1}, x_{2} \in \operatorname{dom}\left(p_{1}\right)$

$$
x_{1} \subseteq x_{2} \Longleftrightarrow p_{1}\left(x_{1}\right) \subseteq p_{1}\left(x_{2}\right) .
$$

However these conditions are not enough. For example, whenever $x \in A_{1}$, $y \in A_{2}$ and $f_{1}(x)=y$, then $f_{0}^{\prime \prime} x=y$, hence $|x|=|y|$ and moreover $\left|A_{0}-x\right|=$ $\left|A_{1}-y\right|$ since $f_{0}$ is a bijection. Similarly for $y, z$ such that $f_{2}(y)=z$. So we need first the following definition:

Definition 2.1 Let $V, W$ be sets such that $|V|=|W|$, and let $X \subseteq \mathcal{P}(V)$ and $Y \subseteq \mathcal{P}(W)$. We say that $X$ and $Y$ are similar and we write $(X)_{V} \sim$ $(Y)_{W}$, or just $X \sim Y$, if there is a bijection $h: V \rightarrow W$ such that $Y=$ $\left\{h^{\prime \prime} x: x \in X\right\}$. Further, if $g$ is a 1-1 mapping such that $\operatorname{dom}(g) \subseteq X$ and $\operatorname{rng}(g) \subseteq Y$, we say that $X$ and $Y$ are similar modulo $g$ and we write $X \sim_{g} Y$, if there is a bijection $h: V \rightarrow W$ such that $Y=\left\{h^{\prime \prime} x: x \in X\right\}$ and $g \subseteq h$.

\footnotetext{
${ }^{4}$ We do not mean necessarily "finite parts", although in many forcing constructions one constructs a generic object from its finite parts, whenever this possible. Here, on the contrary, finiteness would be an obstacle because, as we saw in section 1.1, this property is not absolute for models of TST.
} 
For finite $X, Y$, an equivalent description of the relation $X \sim Y$, can be given as follows. Each finite set $X \subseteq P(V)$ generates a partition of $V$ into sets which are the atoms of the least Boolean algebra that contains $X$. Let $B(X)$ denote this Boolean algebra and let $B_{0}(X)$ denote the set of atoms of $B(X)$. Then the following holds.

Lemma 2.2 Let $V, W$ be sets such that $|V|=|W|$, and let $X \subseteq \mathcal{P}(V)$ and $Y \subseteq \mathcal{P}(W)$ be finite. The following are equivalent:

(a) $X \sim Y$,

(b) $B_{0}(X) \sim B_{0}(Y)$,

(c) There is a bijection $g: B_{0}(X) \rightarrow B_{0}(Y)$ such that $|x|=|g(x)|$ for every $x \in B_{0}(X)$. Of course this bijection can be extended to the whole $B(X)$ with the same property.

Proof. (a) $\Rightarrow(\mathrm{b}):$ Let $X \sim Y$, let $X=\left\{x_{1}, \ldots, x_{n}\right\}$, let $h: V \rightarrow W$ be a bijection such $Y=\left\{h^{\prime \prime} x: x \in X\right\}$, and let $h^{\prime \prime} x_{k}=y_{k}$, for $k=1, \ldots, n$. Therefore $Y=\left\{y_{1}, \ldots y_{n}\right\}$. The elements of $B_{0}(X)$ have the form $\bigcap_{k=1}^{n} x_{k}^{\sigma(k)}$, where $\sigma$ is a mapping $\sigma:\{1, \ldots, n\} \rightarrow\{0,1\}$ and $x_{k}^{\sigma(k)}=x_{k}$ if $\sigma(k)=1$, while $x_{k}^{\sigma(k)}=\overline{x_{k}}=V-x_{k}$ if $\sigma(k)=0$. And similarly for $B_{0}(Y)$. Then for every atom $\bigcap_{k=1}^{n} x_{i}^{\sigma(k)}$ of $B_{0}(X)$ we clearly have $h^{\prime \prime}\left(\bigcap_{k=1}^{n} x_{k}^{\sigma(k)}\right)=\bigcap_{k=1}^{n} h^{\prime \prime} x_{k}^{\sigma(k)}=$ $\bigcap_{k i=1}^{n} y_{k}^{\sigma(k)}$, which is the corresponding atom of $B_{0}(Y)$. Therefore $X \sim Y$ implies $B_{0}(X) \sim B_{0}(Y)$.

(b) $\Rightarrow$ (a) is trivial.

(b) $\Rightarrow(\mathrm{c})$ : Let $B_{0}(X) \sim B_{0}(Y)$, and let $h: V \rightarrow W$ be the bijection such that $B_{0}(Y)=\left\{h^{\prime \prime} x: x \in B_{0}(X)\right\}$. If we set $g(x)=h^{\prime \prime} x$ for every $x \in B_{0}(X)$ then $g: B_{0}(X) \rightarrow B_{0}(Y)$ is a bijection such that $|g(x)|=|x|$.

$(\mathrm{c}) \Rightarrow(\mathrm{b}):$ Let $g: B_{0}(X) \rightarrow B_{0}(Y)$ be a bijection such that $|g(x)|=|x|$. For every $x \in B_{0}(x)$ pick a bijection $h_{x}: x \rightarrow g(x)$. If we set $h=\bigcup_{x \in B_{0}(x)} h_{x}$, $h$ is a bijection between $V$ and $W$ (since the elements of $B_{0}(X)$ form a partition of $V)$ such that $B_{0}(Y)=\left\{h^{\prime \prime}(x): x \in B_{0}(Y)\right\}$ and hence $B_{0}(X) \sim$ $B_{0}(Y)$.

We are now in a position to define coherent pairs.

Definition 2.3 Let $\mathcal{A}=\left(A_{0}, A_{1}, \ldots\right)$ be a model TST. A coherent pair of $\mathcal{A}$ is a pair $p=\left(p_{1}, p_{2}\right)$ of 1-1 mappings with the following properties:

(1) $\operatorname{dom}\left(p_{1}\right) \in A_{2}, r n g\left(p_{1}\right)=\operatorname{dom}\left(p_{2}\right) \in A_{3}$, and $r n g\left(p_{2}\right) \in A_{4}$. We set $u_{1}=\operatorname{dom}\left(p_{1}\right), u_{2}=r n g\left(p_{1}\right)=\operatorname{dom}\left(p_{2}\right)$ and $u_{3}=r n g\left(p_{2}\right)$. 
(2) $p_{1}, p_{2}$ are $\in$-isomorphisms, i.e., for every $x \in u_{1}$ and $y \in u_{2}$,

$$
x \in y \Longleftrightarrow p_{1}(x) \in p_{2}(y) .
$$

(3) $u_{1} \sim u_{2}$ and $u_{2} \sim_{p_{1}} u_{3}$, i.e., there are bijections $g: A_{0} \rightarrow A_{1}$ and $h: A_{1} \rightarrow A_{2}$ such that $u_{2}=\left\{g^{\prime \prime} x: x \in u_{1}\right\}, u_{3}=\left\{h^{\prime \prime} y: y \in u_{2}\right\}$ and $p_{1} \subseteq h$.

For simplicity we often say just "pair" instead of "coherent pair". And instead of $p=\left(p_{1}, p_{2}\right)$ we often write more suggestively

$$
u_{1} \stackrel{p_{1}}{\longrightarrow} u_{2} \stackrel{p_{2}}{\longrightarrow} u_{3} .
$$

Remark 2.4 1) Let

$$
A_{0} \stackrel{f_{0}}{\longrightarrow} A_{1} \stackrel{f_{1}}{\longrightarrow} A_{2} \stackrel{f_{2}}{\longrightarrow} A_{3} \cdots
$$

be an automorphism. It is easy to see that every restriction of $f$ to a finite subset of $A_{1}$ is a coherent pair. Indeed, let $u_{1}$ be a finite subset of $A_{1}$, and let $p_{1}=f_{1}\left\lceil u_{1}, u_{2}=p_{1}^{\prime \prime} u_{1}, p_{2}=f_{2}\left\lceil u_{2}\right.\right.$ and $u_{3}=p_{2}^{\prime \prime} u_{2}$. Consider the pair $u_{1} \stackrel{p_{1}}{\longrightarrow}$ $u_{2} \stackrel{p_{2}}{\longrightarrow} u_{3}$. Since $f_{i+1}=f_{i}^{\prime \prime}$, we have $u_{2}=f_{1}^{\prime \prime} u_{1}=\left\{f_{1}(x): x \in u_{1}\right\}=\left\{f_{0}^{\prime \prime} x:\right.$ $\left.x \in u_{1}\right\}$, hence $u_{1} \sim u_{2}$. Also $u_{3}=f_{2}^{\prime \prime} u_{2}=\left\{f_{2}(y): y \in u_{2}\right\}=\left\{f_{1}^{\prime \prime} y: y \in u_{2}\right\}$ and moreover $p_{1} \subseteq f_{1}$, therefore $u_{2} \sim_{p_{1}} u_{3}$.

2) Condition (3) of the above definition clearly implies that the mappings $p_{1}, p_{2}$ can be extended to isomorphisms between the corresponding Boolean algebras $B\left(u_{i}\right)$ generated by the sets $u_{i}$. So without loss of generality in the above definitions we could take $u_{i}$ to be finite Boolean algebras. (However the converse is false. If $u_{i}$ are Boolean algebras such that $u_{1} \cong u_{2}$ and $u_{2} \cong u_{3}$, then (3) need not be true.)

Notational convention. To facilitate reading, we shall henceforth follow the following convention: The variable $x$ (possibly with subscripts) will range exclusively over the level $A_{1}$ of $\mathcal{A}$, the variable $y$ will range exclusively over $A_{2}$ and the variable $z$ will range exclusively over $A_{3}$.

We define a partial ordering $\leq$ between pairs as follows: If $p=\left(p_{1}, p_{2}\right)$, and $q=\left(q_{1}, q_{2}\right), p \leq q$ iff $p_{1} \supseteq q_{1}$ and $p_{2} \supseteq q_{2}$. In particular, if $u_{1} \stackrel{p_{1}}{\longrightarrow} u_{2} \stackrel{p_{2}}{\longrightarrow}$ $u_{3}$, and $v_{1} \stackrel{q_{1}}{\longrightarrow} v_{2} \stackrel{q_{2}}{\longrightarrow} v_{3}$ are two pairs and $p \leq q$, then for every $i=1,2,3$ $u_{i} \supseteq v_{i}$. 
EXAMPLE 1. The simplest example of a coherent pair is that in which $u_{1}, u_{2}, u_{3}$ are the trivial Boolean subalgebras of $A_{1}, A_{2}, A_{3}$ respectively and $p_{1}, p_{2}$ are the trivial isomorphisms between them. Namely let: $u_{1}=\left\{\emptyset, A_{0}\right\}$, $u_{2}=\left\{\emptyset, A_{1}\right\}, u_{3}=\left\{\emptyset, A_{2}\right\}, p_{i}(\emptyset)=\emptyset$, for $i=1,2, p_{1}\left(A_{0}\right)=A_{1}$, and $p_{2}\left(A_{1}\right)=A_{2}$. So properties (1) and (2) of definition 2.3 are trivially satisfied. Further, since $\mathcal{A}$ is countable, $\left|A_{0}\right|=\left|A_{1}\right|=\left|A_{2}\right|$ in the ground model. Take any bijection $g: A_{0} \rightarrow A_{1}$. Then $g^{\prime \prime} A_{0}=A_{1}$ and $g^{\prime \prime} \emptyset=\emptyset$, that is $u_{2}=\left\{g^{\prime \prime} x: x \in u_{1}\right\}$. Hence $u_{1} \sim u_{2}$. To show that $u_{2} \sim_{p_{1}} u_{3}$, it suffices to find a bijection $h: A_{1} \rightarrow A_{2}$ that extends $p_{1}$, i.e., such that $h(\emptyset)=\emptyset$ and $h\left(A_{0}\right)=A_{1}$. But such a bijection obviously exists: Simply put $h(\emptyset)=\emptyset$, $h\left(A_{0}\right)=A_{1}$ and then extend it on $A_{1}$ arbitrarily.

Example 2. Let $a \in A_{0}$, let $g: A_{0} \rightarrow A_{1}$ be a bijection and let $h$ : $A_{1} \rightarrow A_{2}$ be a bijection such that $h(\emptyset)=\emptyset, h\left(A_{0}\right)=A_{1}, h(\{a\})=\{g(a)\}$ and $h\left(A_{0}-\{a\}\right)=A_{1}-\{g(a\}$. Let also

$u_{1}=\left\{\emptyset, A_{0},\{a\}, A_{0}-\{a\}\right\}$,

$u_{2}=\left\{\emptyset, A_{1},\{g(a)\}, A_{1}-\{g(a)\}\right\}$,

$u_{3}=\left\{\emptyset, A_{2},\{h g(a)\}, A_{2}-\{h g(a)\}\right\}$,

Let $p_{1}: u_{1} \rightarrow u_{2}$ and $p_{2}: u_{2} \rightarrow u_{3}$ be the bijections that preserve the orderings of the elements of $u_{i}$ as cited above. Then $u_{1} \stackrel{p_{1}}{\longrightarrow} u_{2} \stackrel{p_{2}}{\longrightarrow} u_{3}$ is a coherent pair. Indeed $u_{2}=\left\{g^{\prime \prime} x: x \in u_{1}\right\}, u_{3}=\left\{h^{\prime \prime} y: y \in u_{2}\right\}$ and $p_{1} \subseteq h$. Hence $u_{1} \sim u_{2}$ and $u_{2} \sim_{p_{1}} u_{3}$. Finally it is easy to check that $p=\left(p_{1}, p_{2}\right)$ is an $\in$-automorphism.

What kind of object is a coherent pair $p=\left(p_{1}, p_{2}\right)$ with respect to the model $\mathcal{A}$ ? By definition $u_{1} \in A_{2}, u_{2} \in A_{3}$ and $u_{3} \in A_{4}$. But $p_{1}, p_{2}$ need not be coded in $\mathcal{A}$, when they are infinite. Moreover, the property of coherence requires the existence of bijections $g: A_{0} \rightarrow A_{1}$ or $h: A_{1} \rightarrow A_{2}$. By lemma 1.3, no bijection $g: A_{0} \rightarrow A_{1}$ or $h: A_{1} \rightarrow A_{2}$ can be definable in $\mathcal{A}$ (and hence be coded by an object in $\mathcal{A}$ ). Therefore $g, h$ necessarily, and $p_{1}, p_{2}$ possibly, will be external objects for $\mathcal{A}$, existing only for an observer in the ground model $M$, so we can't speak about them in $L_{\mathrm{TST}}$.

In order to be able to speak about coherent pairs formally, we need to extend the language $L_{\mathrm{TST}}$ by adding new untyped set variables, denoted by lower case Greek letters $\alpha, \beta, \ldots$, intended to range over unstratified objects of models of TST. These unstratified objects will include infinite mappings between distinct levels of $\mathcal{A}$.

To motivate the definitions, given a model $\mathcal{A}=\left(A_{0}, A_{1}, \ldots\right)$ of TST, 
define (in the ground model $M$ ) the countable cumulative hierarchy over $A_{0}$, denoted by $V_{\omega}\left(A_{0}\right)$, as usual by:

$$
\begin{aligned}
& V_{0}\left(A_{0}\right)=A_{0} . \\
& V_{n+1}\left(A_{0}\right)=V_{n}\left(A_{0}\right) \cup \mathcal{P}\left(V_{n}\left(A_{0}\right)\right) . \\
& V_{\omega}=\bigcup_{n \in \mathbb{N}} V_{n}\left(A_{0}\right) .
\end{aligned}
$$

Since for every $n, A_{n+1} \subseteq \mathcal{P}\left(A_{n}\right)$, it is easy to check by induction that $A_{n} \subseteq V_{n}\left(A_{0}\right)$ for all $n \in \mathbb{N}$. Let us call the elements of $V_{\omega}\left(A_{0}\right)$ complex, in contrast to the elements of $\bigcup_{n} A_{n}$ which are the stratified ones. For example a bijection between $A_{i}$ and $A_{i+1}$ is a complex object.

Note that according to the above definition each stratified set is already complex. So the relation of complex sets to stratified ones is similar to the relation between classes and sets in the standard theory of classes. Each set is a class but not the other way around. So as we speak of proper classes, we shall speak here of proper complex sets. Also as the variable $X$ in class theory ranges over classes in general (including sets), while $x$ ranges over sets only, similarly here $\alpha$ ranges over complex sets in general (including stratified ones), while $x^{i}$ ranges over stratified sets only.

Let $L_{\mathrm{TST}^{c}}$ be the new language containing the variables $\alpha$, besides $x^{i}$. The formulas of $L_{\mathrm{TST}^{c}}$ are those of $L_{\mathrm{TST}}$ plus formulas of the form $\alpha \varepsilon \beta$, $x^{i} \varepsilon \alpha, \alpha=\beta$. A formula of $L_{\mathrm{TST}^{c}}$ is said to be stratified if it is a formula of $L_{\mathrm{TST}}$.

Let $\mathrm{TST}^{c}$ be the theory in the language $L_{\mathrm{TST}^{c}}$ with axioms ${ }^{5}$ :

(I) Comprehension for stratified formulas. (This is just the corresponding axiom (Co) of TST.)

(II) Every stratified set is complex:

$$
\forall x^{i} \exists \alpha\left(x^{i}=\alpha\right) .
$$

(III) Elements of stratified sets are stratified:

$$
\forall \alpha\left(\alpha \varepsilon x^{i+1} \Rightarrow \exists x^{i}\left(x^{i}=\alpha\right)\right) .
$$

\footnotetext{
${ }^{5}$ The theory $\mathrm{TST}^{c}$, as we use it here, is only a temporary tool for the proof of the main theorem, with no further significance. That is why we do not specify any particular kinds of complex objects that could exist in the theory. I.e., we do not include any comprehension scheme for complex objects. Perhaps a more specific and refined extension of TST, with an interest in itself, might be considered and studied.
} 
(IV) Extensionality:

$$
\forall \gamma(\gamma \varepsilon \alpha \Leftrightarrow \gamma \varepsilon \beta) \Rightarrow \alpha=\beta .
$$

In view of axioms (II) and (III), it is clear that (IV) extends the axiom (Ex) of TST, so $\mathrm{TST}^{c}$ is an extension of the theory TST.

Standard models of $\operatorname{TST}^{c}$ have the form $(\mathcal{A}, C)=\left(A_{0}, A_{1}, \ldots, C\right)$, where $A_{i}$ contain the stratified sets as usual, and $C$ contains the proper complex objects. Natural such models are e.g. those of the form

$$
\left(X, \mathcal{P}(X), \mathcal{P}^{2}(X), \ldots, C\right)
$$

where $\left.C=V_{\omega}(X)-\bigcup_{n} \mathcal{P}^{n}(X)\right)$.

In $\mathrm{TST}^{c}$, ordered pairs, relations, functions etc. are defined as usual.

Lemma 2.5 The property " $\alpha$ is a coherent pair" is definable in $\mathrm{TST}^{c}$.

Proof. The property " $\alpha$ is a coherent pair" is the conjunction of the following statements:

(1) $\alpha$ is a pair of objects $\left(\alpha_{1}, \alpha_{2}\right)$ each of which is a 1-1 mapping, such that for some $x_{1}^{2}, x_{2}^{3}, x_{3}^{4}$, dom $\left(\alpha_{1}\right)=x_{1}^{2}, \operatorname{rng}\left(\alpha_{1}\right)=\operatorname{dom}\left(\alpha_{2}\right)=x_{2}^{3}$ and $r n g\left(\alpha_{2}\right)=x_{3}^{4}$.

(2) $\alpha_{1}$ and $\alpha_{2}$ are $\varepsilon$ - and $\subseteq$-preserving mappings.

(3) There are bijections $\gamma_{1}: A_{1} \rightarrow A_{2}$ and $\gamma_{2}: A_{2} \rightarrow A_{3}$ which entail that $x_{1}^{2} \sim x_{2}^{3}$ and $x_{2}^{3} \sim_{\alpha_{1}} x_{3}^{4}$.

The statements " $\gamma_{1}: A_{1} \rightarrow A_{2}$ is a bijection" and " $\gamma_{2}: A_{2} \rightarrow A_{3}$ is bijection" are clearly expressible in $L_{\mathrm{TST}^{c}}$.

\section{$2.2 \quad$ Extendibility}

Recall that if $P$ is the set of finite 1-1 mappings from a set $A$ into a set $B$, ordered by (reverse) inclusion, and $G$ is a generic subset of $P$, in order to prove that $f=\cup\{p: p \in G\}$ is a bijection between $A$ and $B$, we need to show that for every $p \in P$ and every $a \in A$ we can extend $p$ to a condition $q$ such that $a \in \operatorname{dom}(q)$ and/or $a \in r n g(q)$. This is the extendibility property of $P$. Extendibility guarantees that the sets of conditions $q$ such that $a \in \operatorname{dom}(q)$ or $a \in r n g(g)$ are all dense in $P$, and hence meet $G$. For the $P$ of the example just mentioned the property holds trivially. However for the set of coherent pairs extendibility is not a simple matter to hold. As follows from Example 3 below, a lot of coherent pairs are not extendible. 
Definition 2.6 Let $p=u_{1} \stackrel{p_{1}}{\longrightarrow} u_{2} \stackrel{p_{2}}{\longrightarrow} u_{3}$ be a pair. We say that $p$ is everywhere extendible or just extendible if for every $t \in A_{1} \cup A_{2} \cup A_{3}$, there is a pair $v_{1} \stackrel{q_{1}}{\longrightarrow} v_{2} \stackrel{q_{2}}{\longrightarrow} v_{3}$ such that $q \leq p$ and $t \in v_{1} \cup v_{2} \cup v_{3}$. When such a pair $q=\left(q_{1}, q_{2}\right)$ exists, we say for simplicity that $q$ captures $t$.

Definition 2.7 Let $p=u_{1} \stackrel{p_{1}}{\longrightarrow} u_{2} \stackrel{p_{2}}{\longrightarrow} u_{3}$ be a pair. $p$ is said to be 1 extendible if it is extendible. $p$ is said to be $(n+1)$-extendible if for every $t \in A_{1} \cup A_{2} \cup A_{3}$ there is a pair $q=\left(q_{1}, q_{2}\right)$ such that $q$ captures $t, q \leq p$ and $q$ is $n$-extendible. $p$ is said to be $\omega$-extendible if it is $n$-extendible for all $n \geq 1$.

An easy induction shows that if $\left(p_{1}, p_{2}\right)$ is $n$-extendible and $m<n$, then $\left(p_{1}, p_{2}\right)$ is $m$-extendible.

ExAmple 3. Consider the coherent pair of Example 2, where

$u_{1}=\left\{\emptyset, A_{0},\{a\}, A_{0}-\{a\}\right\}$,

$u_{2}=\left\{\emptyset, A_{1},\{g(a)\}, A_{1}-\{g(a)\}\right\}$,

$u_{3}=\left\{\emptyset, A_{2},\{h g(a)\}, A_{2}-\{h g(a)\}\right\}$.

If we choose the bijections $g, h$ so that $|g(a)| \neq|h g(a)|$, then we can easily see that the pair $\left(p_{1}, p_{2}\right)$ is not extendible. Indeed, first note that $h g(a) \notin u_{2}$. For if $h g(a) \in u_{2}$, then, because of the coherence, $g(a) \in u_{1}$ and $p_{1}(g(a))=h g(a)$, hence $|g(a)|=|h g(a)|$, a contradiction. Therefore $h g(a) \notin u_{2}$. By extendibility, there must be a pair $q=\left(q_{1}, q_{2}\right)$ such that $q \leq p$ and $q$ captures $h g(a)$. But $h g(a)$ is the unique element of an element of $u_{3}$, namely $\{h g(a)\}$. Therefore, in order for $q$ to be coherent, it must contain the corresponding unique element $g(a)$ of $\{g(a)\}$ of $u_{2}$ and moreover $q_{1}(g(a))=h g(a)$. But then also we must have $|g(a)|=|h g(a)|$, which is not the case. Thus $p$ is not extendible.

Theorem 2.8 (MAIn TheOREM) Let $M$ be a countable model of ZFC in which for every $n \in \mathbb{N}$, there is a s.t. model $\mathcal{A}$ of TST that contains an $n$-extendible coherent pair. Then there is a generic extension $M[G]$ of $M$ that contains a model of $\mathrm{NF}$. Conversely, if $M$ contains a model of $\mathrm{NF}$, then in $M$ there is a s.t. model $\mathcal{A}$ of TST that contains an n-extendible pair, for every $n \geq 1$.

Proof. Let us show first the converse. Let $M$ contain a model of NF. Then $M$ contains also a model $\mathcal{A}=\left(A_{0}, A_{1}, A_{2}, \ldots, R\right)$ of TST with an 
automorphism

$$
A_{0} \stackrel{f_{0}}{\longrightarrow} A_{1} \stackrel{f_{1}}{\longrightarrow} A_{2} \stackrel{f_{2}}{\longrightarrow} A_{3} \cdots
$$

By lemma 1.6 we may assume that $\mathcal{A}$ is s.t. As we saw in remark 2.4 (1), if $u_{1} \subseteq A_{1}$ is finite, and we set $p_{1}=f_{1}\left\lceil u_{1}, u_{2}=p_{1}^{\prime \prime} u_{1}, p_{2}=f_{2}\left\lceil u_{2}\right.\right.$ and $u_{3}=p_{2}^{\prime \prime} u_{2}$, then

$$
p=u_{1} \stackrel{p_{1}}{\longrightarrow} u_{2} \stackrel{p_{2}}{\longrightarrow} u_{3}
$$

is a coherent pair. Moreover $p$ is $n$-extendible for every $n \geq 1$ (that is, $\omega$-extendible). This can be shown inductively for all such restrictions $p$ of $f$. Indeed, obviously all such restrictions $p$ are extendible, i.e., 1-extendible. Suppose all such $p$ are $n$-extendible and pick some specific $p$. If $t \in A_{1} \cup A_{2} \cup$ $A_{2}$, then we can choose a part $q$ of $f$ which extends $p$ and captures $t$. Since by assumption $q$ is $n$-extendible, $p$ is $(n+1)$-extendible.

We come to the converse. Let $M$ be a countable model of ZFC such that for every $n \in \mathbb{N}$, there is a s.t. model $\mathcal{A}$ of TST in $M$ that contains an $n$-extendible coherent pair. We turn to the language of $\mathrm{TST}^{c}$. By lemma 2.5 , the property " $\alpha$ is a coherent pair" is definable in $\operatorname{TST}^{c}$. Let $\theta_{0}(\alpha)$ be the formula that defines it. It is easy to see by induction on $n$, that for every $n \geq 1$ there is a formula $\theta_{n}(\alpha)$ of $L_{\mathrm{TST}^{c}}$ expressing the fact that " $\alpha$ is an $n$-extendible coherent pair". Recall that a coherent pair is of the form $p=u_{1} \stackrel{p_{1}}{\longrightarrow} u_{2} \stackrel{p_{2}}{\longrightarrow} u_{3}$ with $u_{i} \in A_{i+1}$, i.e., $u_{i}$ is of type $i+1$. So using typed variables in the place of $u_{i}$ and untyped variables in the place of $p_{i}$ and $p$, a coherent pair is more formally written as $\alpha=x_{1}^{2} \stackrel{\alpha_{1}}{\longrightarrow} x_{2}^{3} \stackrel{\alpha_{2}}{\longrightarrow} x_{3}^{4}$.

Now suppose that $\theta_{n}(\alpha)$ is defined for $n \geq 0$, where,

$$
\alpha=x_{1}^{2} \stackrel{\alpha_{1}}{\longrightarrow} x_{2}^{3} \stackrel{\alpha_{2}}{\longrightarrow} x_{3}^{4} .
$$

Then $\theta_{n+1}(\alpha)$ is defined to be the formula:

$$
\bigwedge_{i=1}^{3}\left[\forall z^{i} \exists y_{1}^{2} y_{2}^{3} y_{3}^{4} \exists \beta_{1} \beta_{2} \exists \beta\left(\beta=y_{1}^{2} \stackrel{\beta_{1}}{\longrightarrow} y_{2}^{3} \stackrel{\beta_{2}}{\longrightarrow} y_{3}^{4} \& \theta_{n}(\beta) \& \beta \leq \alpha \& z^{i} \varepsilon y_{i}^{i+1}\right)\right] .
$$

It is easy to check that $\theta_{n+1}(\alpha)$ expresses the fact that $\alpha$ is an $(n+1)$ extendible coherent pair. Moreover, by induction we easily check that for $m<n$, every $n$-extendible coherent pair is $m$-extendible, therefore

$$
m<n \& \theta_{n}(\alpha) \Rightarrow \theta_{m}(\alpha) .
$$

Consider the theory

$$
T=\operatorname{TST}^{c}+\left\{\theta_{n}(b): n \in \mathbb{N}\right\}
$$


in the language $L_{\mathrm{TST}^{c}}(b)$ with a new constant $b$. By our assumption, for every $n \in \mathbb{N}$, $\operatorname{TST}^{c}+\theta_{n}(b)$ has a model, so by (5) and compactness $T$ has a model. Let $(\mathcal{A}, C)$ be a countable model of $T$ in $M$. Already $(\mathcal{A}, C)$ contains an $\omega$-extendible element (the interpretation of the constant $b$ in $(\mathcal{A}, C)$ ). Let $(\mathcal{B}, D) \in M$ be a saturated model such that $(\mathcal{A}, C) \preceq(\mathcal{B}, D)$. (As to the kind of saturation, $\omega$-saturation and even recursive saturation suffices.) $(\mathcal{B}, D) \models T$, so $(\mathcal{B}, D)$ also contains $\omega$-extendible pairs. Let $P_{\omega}$ be the set of $\omega$-extendible coherent pairs of $(\mathcal{B}, D)$. Clearly $P_{\omega} \in M$ and we use $\left(P_{\omega}, \leq\right)$ as a set of forcing conditions in $M$.

Claim 1: Let $\mathcal{B}=\left(B_{0}, B_{1}, B_{2}, \ldots\right)$. If $p \in P_{\omega}$ and $t \in B_{1} \cup B_{2} \cup B_{3}$, then there is a $q \in P_{\omega}$ such that $q \leq p$ and $q$ captures $t$.

Proof. By assumption, $p$ is $\omega$-extendible, i.e., $n$-extendible for every $n \geq 1$. Therefore, given $t \in B_{1} \cup B_{2} \cup B_{3}$ for each $n$, there is an $n$-extendible pair $q_{n}$ such that $q_{n} \leq p$ and $q_{n}$ captures $t$. Consider the type

$$
s(\alpha)=\left\{\alpha \leq p \quad \& \alpha \text { captures } t \quad \& \theta_{n}(\alpha): n \in \mathbb{N}\right\}
$$

in the language $L_{\mathrm{TST}^{c}}$. Then $s(\alpha)$ is finitely satisfiable in $(\mathcal{B}, D)$ (moreover $s(\alpha)$ is a recursive type, if one wants to use recursive saturation). Therefore it is satisfiable in $(\mathcal{B}, D)$, and hence there is a $q \in P_{\omega}$ such that $q \leq p$ and $q$ captures $t$. This proves Claim 1 .

Claim 2. Let $G$ be a generic subset of $P_{\omega}$. Then $G$ defines a pair of bijections $B_{1} \stackrel{f_{1}}{\longrightarrow} B_{2} \stackrel{f_{2}}{\longrightarrow} B_{3}$ that satisfies conditions (3) and (4).

Proof. Obviously if $f_{1}=\bigcup\left\{p_{1}: p \in G\right\}$ and $f_{2}=\bigcup\left\{p_{2}: p \in G\right\}$, then $\left(f_{1}, f_{2}\right)$ is a pair of $\in$ - and $\subseteq$-preserving mappings. So it suffices to see that $\operatorname{dom}\left(f_{1}\right)=B_{1}, \operatorname{dom}\left(f_{2}\right)=r n g\left(f_{1}\right)=B_{2}$ and $r n g\left(f_{2}\right)=B_{3}$. But this follows immediately from Claim 1 (and genericity). This proves Claim 2.

Now in the generic extension $M[G]$ of $M, \mathcal{B}$ is still a model of TST, since the sentences " $\mathcal{B} \models \phi$ ", for $\phi \in L_{\mathrm{TST}}$, are $\Delta_{0}$ (since $\mathcal{B} \in M$ ) and hence absolute between $M$ and $M[G]$. Therefore in $M[G], \mathcal{B}$ is a model of TST, whose part $\left(B_{1}, B_{2}, B_{3}\right)$, by Claim 2 , has a pair of bijections $\left(f_{1}, f_{2}\right)$ satisfying $(3)$ and (4). Thus, by lemma $1.9, \mathcal{B}$ yields a model of NF in $M[G]$.

After theorem 2.8, the first task is to find models of TST containing extendible (i.e., 1-extendible) pairs. The most natural candidate pair to be 
extendible would be the pair of Example 1. Given the model $\mathcal{A}$, let us denote it by $o^{\mathcal{A}}$, i.e.,

$$
o^{\mathcal{A}}:=\mathbf{2}_{1} \stackrel{o_{1}}{\longrightarrow} \mathbf{2}_{2} \stackrel{o_{2}}{\longrightarrow} \mathbf{2}_{3},
$$

where $\boldsymbol{2}_{i}$ denotes the trivial Boolean subalgebra of $A_{i}, i=1,2,3$, and $o_{1}, o_{2}$ are the mappings such that $o_{i}(\emptyset)=\emptyset$ and $o_{i}\left(A_{i-1}\right)=A_{i}$. So the question becomes: Are there $\mathcal{A}$ such that $o^{\mathcal{A}}$ is extendible in $\mathcal{A}$ ? Of course crucial for the extendibility of $o^{\mathcal{A}}$ will be the properties of the underlying model $\mathcal{A}$. We shall show in the next section that for $\mathcal{A}$ satisfying some rather mild conditions, $O^{\mathcal{A}}$ is 1-extendible. Moreover, the main theorem above can be equivalently formulated as follows:

Theorem 2.9 (MAin TheOREM) Let $M$ be a countable model of ZFC in which for every $n \in \mathbb{N}$, there is a s.t. model $\mathcal{A}$ of TST such that $o^{\mathcal{A}}$ is $n$ extendible. Then there is a generic extension $M[G]$ of $M$ that contains a model of NF. Conversely, if $M$ contains a model of NF, then there is a s.t. model $\mathcal{A} \in M$ such that $\mathcal{O}^{\mathcal{A}}$ is $n$-extendible, for every $n \geq 1$.

\section{Existence of 1-extendible pairs}

The property of 1-extendibility (or just extendibility) of a pair $\left(p_{1}, p_{2}\right)$ in $\mathcal{A}$ splits into three subproperties, namely $A_{i}$-extendibility, for $i=1,2,3$. Specifically $p=\left(p_{1}, p_{2}\right)$ is said to be $A_{i}$-extendible, for $i=1,2,3$, if for every $t \in A_{i}$ there is a pair $q=\left(q_{1}, q_{2}\right)$ such that $q \leq p$ and $q$ captures $t$. So

$$
p \text { is extendible } \Longleftrightarrow \bigwedge_{i=1}^{3}\left(p \text { is } A_{i} \text {-extendible }\right) \text {. }
$$

Recall from section 1.1 that Fin $(x)$ denotes the property of internal finiteness, while " $x$ is finite" means that $x$ is finite in the ground model.

Definition 3.1 A model $\mathcal{A}$ of TST is called regular if for every $x \in A$,

$$
x \text { is finite } \Longleftrightarrow \mathcal{A}=\operatorname{Fin}(x) .
$$

Another property we shall need concerning the model $\mathcal{A}$ is the property of "richness" used already by Grishin in [2]. (T. Forster [1], p. 60, calls the property "saturation in the sense of Grishin". Since saturation has several other meanings, we prefer a more neutral name.) 
Definition 3.2 The Boolean algebra $A_{i+1}$ is said to be rich if for every infinite (with respect to the ground model) $x \in A_{i+1}$, there is a $x_{1} \in A_{i+1}$ such that $x_{1} \subseteq x$ and both $x_{1}$ and $x-x_{1}$ are infinite. The structure $\mathcal{A}$ is said to be rich if every level $A_{i+1}$, for $i \geq 0$, is rich.

If $\mathcal{A}$ is regular, then the property of richness is definable in $\mathcal{A}$. Moreover the following holds:

Lemma 3.3 Let $\langle\langle X\rangle\rangle$ be a full model of TST and let $\mathcal{A}$ be the level collapse of an elementary submodel of $\langle\langle X\rangle\rangle$. Then $\mathcal{A}$ is (a) regular and (b) rich.

Proof. Let $\mathcal{B} \preceq\langle\langle X\rangle\rangle$ and let $\mathcal{A}=l c(\mathcal{B})$. Let $\sigma: \mathcal{B} \rightarrow \mathcal{A}$ be the level collapsing function.

(a) Let $A_{k}$ be the levels of $\mathcal{A}$ and $B_{k}$ the levels of $\mathcal{B}$. Let $x \in A_{i+1}$. We have to show that " $x$ is finite" $\Longleftrightarrow \mathcal{A} \models \operatorname{Fin}(x)$.

By (1) of section 1.1, " $x$ is finite" $\Rightarrow \mathcal{A}=\operatorname{Fin}(x)$ is always true.

So suppose $\mathcal{A} \models \operatorname{Fin}(x)$. Let $x=\sigma(y)$, for some $y \in B_{i+1}$. Now $\operatorname{Fin}(\cdot)$ is a formula of $L_{\mathrm{TST}}$, so by lemma 1.2 ,

$$
\mathcal{A}=\operatorname{Fin}(x) \Longleftrightarrow \operatorname{lc}(\mathcal{B}) \models \operatorname{Fin}(\sigma(y)) \Longleftrightarrow \mathcal{B}=\operatorname{Fin}(y)
$$

Consequently, since $\mathcal{B} \preceq\langle\langle X\rangle\rangle,\langle\langle X\rangle\rangle \models$ Fin $(y)$. The latter clearly implies that " $y$ is finite". Now $\mathcal{B}$ is standard and $\sigma(y)=\left\{\sigma(u): u \in y \cap B_{i}\right\}$. Therefore $x=\sigma(y)$ is finite, since $y \cap B_{i}$ is so.

(b) In view of regularity and the absoluteness of the property " $x$ is finite", the property of richness is expressed by the sentence of $L_{\mathrm{TST}}$ (written without type indicators):

$$
\phi: \forall x\left[\neg \operatorname{Fin}(x) \Rightarrow \exists x_{1} x_{2}\left(\neg F i n\left(x_{1}\right) \wedge \neg F\left(x_{2}\right) \wedge x=x_{1} \cup x_{2} \wedge x_{1} \cap x_{2}=\emptyset\right)\right] .
$$

Since obviously $\langle\langle X\rangle\rangle \models \phi$, it follows that $\mathcal{B} \models \phi$ and, by $1.2, \mathcal{A} \models \phi$.

\section{1 $A_{1}$ - and $A_{2}$-extendibility}

For every $x \in A_{1}$ (resp. $y \in A_{2}, z \in A_{3}$ ) we shall write for convenience $-x$ (resp. $-y,-z$ ) instead of $A_{0}-x$ (resp. $A_{1}-y, A_{2}-z$ ).

Lemma 3.4 Let $\mathcal{A}$ be a countable rich model of TST. Then the pair $o^{\mathcal{A}}$ is $A_{1}$ - and $A_{2}$-extendible in $\mathcal{A}$. 
Proof. We have $o^{\mathcal{A}}=\mathbf{2}_{1} \stackrel{o_{1}}{\longrightarrow} \mathbf{2}_{2} \stackrel{o_{2}}{\longrightarrow} \mathbf{2}_{3}$, where $\mathbf{2}_{1}=\left\{\emptyset, A_{0}\right\}, \mathbf{2}_{2}=\left\{\emptyset, A_{1}\right\}$, $\mathbf{2}_{3}=\left\{\emptyset, A_{2}\right\}$, and $o_{i}(\emptyset)=\emptyset$, for $i=1,2, o_{1}\left(A_{0}\right)=A_{1}, o_{2}\left(A_{1}\right)=A_{2}$.

$A_{1}$-Extendibility:

Pick first any $x_{0} \in A_{1}$, hence $x_{0} \subseteq A_{0}$. We have to find $y_{0} \subseteq A_{2}$ and $z_{0} \subseteq A_{3}$ such that, if

$$
\begin{aligned}
& v_{1}=\left\{A_{0}, \emptyset, x_{0},-x_{0}\right\}, \\
& v_{2}=\left\{A_{0}, \emptyset, y_{0},-y_{0}\right\}, \\
& v_{3}=\left\{A_{0}, \emptyset, z_{0},-z_{0}\right\},
\end{aligned}
$$

and if $q_{1}: v_{1} \rightarrow v_{2}, q_{2}: v_{2} \rightarrow v_{3}$ are the bijections that preserve the orderings of the elements of $v_{i}$ as cited above, then $\left(q_{1}, q_{2}\right)$ is coherent pair.

First, because of the richness of $\mathcal{A}$, we can choose $y_{0} \in A_{2}$ such that

(i) $\left|y_{0}\right|=\left|x_{0}\right|$ and $\left|-y_{0}\right|=\left|-x_{0}\right|$.

This guarantees that $v_{1} \sim v_{2}$. Then, we need a $z_{0} \in A_{3}$ such that

(ii) $\left|z_{0}\right|=\left|y_{0}\right|$ and $\left|-z_{0}\right|=\left|-y_{0}\right|$ and moreover

(a) $\emptyset \in y_{0} \Longleftrightarrow \emptyset \in z_{0}$,

(b) $A_{0} \in y_{0} \Longleftrightarrow A_{1} \in z_{0}$,

(c) $x_{0} \in y_{0} \Longleftrightarrow y_{0} \in z_{0}$,

(d) $-x_{0} \in y_{0} \Longleftrightarrow-y_{0} \in z_{0}$.

The above conditions (i), (ii) and (a)-(d) suffice for $\left(q_{1}, q_{2}\right)$ to be a coherent pair. Indeed, because of (a)-(d), $\left(q_{1}, q_{2}\right)$ is an $\in$-automorphism. It remains to show that $v_{2} \sim_{q_{1}} v_{3}$. For that it suffices to construct a bijection $h: A_{1} \rightarrow A_{2}$ such that $h^{\prime \prime} y_{0}=z_{0}, h^{\prime \prime}-y_{0}=-z_{0}$, and $h(\emptyset)=\emptyset, h\left(A_{1}\right)=A_{2}$, $h\left(x_{0}\right)=y_{0}$ and $h\left(-x_{0}\right)=-y_{0}$. But this obviously possible because of (ii) and (a)-(d).

Now, the existence of a $z_{0} \in A_{3}$ such that (ii) and (a)-(d) hold follows easily from the fact that $\mathcal{A}$ is rich. We just find $z_{0}$ so that (ii) holds and in addition $z_{0}$ skips or contains each of the four elements $\emptyset, A_{1}, y_{0},-y_{0}$, accordingly when $y_{0}$ does so with respect to the corresponding element of $v_{1}$.

$A_{2}$-Extendibility:

Let $y_{0} \in A_{2}$, i.e, $y_{0} \subseteq A_{1}$. Using the richness of $\mathcal{A}$, we can choose a set $x_{0} \in A_{1}$ such that $\left|x_{0}\right|=\left|y_{0}\right|$ and $\left|-x_{0}\right|=\left|-y_{0}\right|$. Now we are as in the first step of $A_{1}$-extendibility. We continue and find $z_{0}$ precisely as we did there. $\dashv$ 


\section{2 $\quad A_{3}$-Extendibility}

The case of $A_{3}$-extendibility of $o^{\mathcal{A}}$ is harder.

Lemma 3.5 Let $\langle\langle D\rangle\rangle$ be a full model of TST (with infinite $D$ ) and let $\mathcal{A}=l c(\mathcal{B})$ for some countable $\mathcal{B} \preceq\langle\langle D\rangle\rangle$. Then the pair $o^{\mathcal{A}}$ is $A_{3}$-extendible in $\mathcal{A}$.

Proof. Given $z_{0} \in A_{3}$ we have to show that there are $x \in A_{1}$ and $y \in A_{2}$ such that the following conditions, isolated above, in the case of $A_{1}$-extendibility, hold simultaneously:

(i) $|y|=|x|$ and $|-y|=|-x|$,

(ii) $\left|z_{0}\right|=|y|$ and $\left|-z_{0}\right|=|-y|$,

(a) $\emptyset \in y \Longleftrightarrow \emptyset \in z_{0}$,

(b) $A_{0} \in y \Longleftrightarrow A_{1} \in z_{0}$,

(c) $x \in y \Longleftrightarrow y \in z_{0}$,

(d) $-x \in y \Longleftrightarrow-y \in z_{0}$.

For $x \in A_{1}, y \in A_{2}, z \in A_{3}$ let us write $\operatorname{Sim}(x, y)$ if $|x|=|y|$ and $|-x|=$ $|-y|$, and similarly for $\operatorname{Sim}(y, z)$. We shall first argue by contradiction, assuming that $o^{\mathcal{A}}$ is not extendible ate $z_{0}$, and hence there are no $x \in A_{1}$ and $y \in A_{2}$ such that (i), (ii) and (a)-(d) hold simultaneously. Then the following is true:

$$
\begin{array}{r}
\left(\forall x \in A_{1}\right)\left(\forall y \in A_{2}\right)\left[\operatorname{Sim}(x, y) \& \operatorname{Sim}\left(y, z_{0}\right) \Rightarrow\left(\emptyset \in y \nLeftarrow \emptyset \in z_{0}\right) \vee\right. \\
\left(A_{0} \in y \nLeftarrow A_{1} \in z_{0}\right) \vee \\
\left(x \in y \nLeftarrow y \in z_{0}\right) \vee \\
\left.\left(-x \in y \nLeftarrow-y \in z_{0}\right)\right] .
\end{array}
$$

Now since $z_{0}$ is given, one of the following is the case:

$\emptyset \in z_{0} \& A_{1} \in z_{0}$

$\emptyset \notin z_{0} \& A_{1} \in z_{0}$

$\emptyset \in z_{0} \& A_{1} \notin z_{0}$, 
$\emptyset \notin z_{0} \& A_{1} \notin z_{0}$.

Without serious loss of generality (as will be evident below), we shall assume that $\emptyset \in z_{0}$ and $A_{1} \in z_{0}$, or $\left\{\emptyset, A_{1}\right\} \subseteq z_{0}$. Then we have to search for $y \in A_{2}$ such that $\left\{\emptyset, A_{0}\right\} \subseteq y$. In view of this restriction (8) transforms to the following:

$$
\begin{array}{r}
\left(\forall x \in A_{1}\right)\left(\forall y \in A_{2}\right)\left[\operatorname{Sim}(x, y) \& \operatorname{Sim}\left(y, z_{0}\right) \&\left\{\emptyset, A_{0}\right\} \subseteq y \Rightarrow\right. \\
\left(x \in y \nLeftarrow y \in z_{0}\right) \vee \\
\left.\left(-x \in y \nLeftarrow-y \in z_{0}\right)\right] .
\end{array}
$$

Case 1. Suppose that $z_{0}$ is finite. Pick some $y_{0} \in A_{2}$ such that $\operatorname{Sim}\left(y_{0}, z_{0}\right),\left\{\emptyset, A_{0}\right\} \subseteq y_{0}$ and $y_{0} \notin z_{0}$ and $-y_{0} \notin z_{0}$. Since $z_{0}$ is finite this choice of $y_{0}$ is obviously possible. Then it follows from (9) that for every $x$ such that $\operatorname{Sim}\left(x, y_{0}\right)$, it must be $x \in y_{0}$ or $-x \in y_{0}$. But clearly there are infinitely many such $x$, so $y_{0}$ must be infinite, contrary to the assumption that $\left|y_{0}\right|=\left|z_{0}\right|=$ finite.

Case 2. Suppose that $z_{0}$ is cofinite. Pick a $x_{0} \in A_{1}$ such that $\operatorname{Sim}\left(x_{0}, z_{0}\right)$. Let

$$
Y=\left\{y \in A_{2}: \operatorname{Sim}\left(y, z_{0}\right) \wedge\left\{\emptyset, A_{0}\right\} \subseteq y \wedge x_{0} \in y \wedge-x_{0} \in y\right\} .
$$

Then it follows from (9) that $\forall y \in Y\left(y \notin z_{0} \vee-y \notin z_{0}\right)$. That is, $\forall y \in$ $Y\left(y \in-z_{0} \vee-y \in-z_{0}\right)$. But $Y$ is infinite, while $-z_{0}$ is finite. This is a contradiction.

Case 3. Suppose finally that $z_{0}$ and $-z_{0}$ are infinite. Since $z_{0}$ and $-z_{0}$ are countable this amounts to $\left|z_{0}\right|=\left|-z_{0}\right|$. Let us call such sets "uniform". Let $A_{1}^{u}=\left\{x \in A_{1}:|x|=|-x|\right\}$ and $A_{2}^{u}=\left\{y \in A_{2}:|y|=|-y|\right\}$ be the sets of "uniform" sets in $A_{1}$ and $A_{2}$ respectively. Since $\mathcal{A}=\pi^{\prime \prime} \mathcal{A}^{\prime} \preceq\langle\langle D\rangle\rangle$, by lemma $3.3, \mathcal{A}$ is regular. So " $x$ is infinite" $\Longleftrightarrow \mathcal{A} \models \neg F i n(x)$. By countability and regularity,

$$
\begin{aligned}
&|x|=|-x| \Longleftrightarrow x \text { is infinite } \wedge-x \text { is infinite } \Longleftrightarrow \\
& \mathcal{A} \models \neg \operatorname{Fin}(x) \wedge \neg \operatorname{Fin}(-x) .
\end{aligned}
$$

Therefore the sets $A_{1}^{u}$ and $A_{2}^{u}$ are definable in $\mathcal{A}$. Moreover, since $\left|z_{0}\right|=\left|-z_{0}\right|$, the condition $\operatorname{Sim}(x, y) \wedge \operatorname{Sim}\left(y, z_{0}\right)$ in the formula (9) is equivalent to $x \in A_{1}^{u} \wedge y \in A_{2}^{u}$. So (9) is written more simply 


$$
\begin{aligned}
&\left(\forall x \in A_{1}^{u}\right)\left(\forall y \in A_{2}^{u}\right)\left[\left\{\emptyset, A_{0}\right\} \subseteq y\right. \Rightarrow \\
&\left(x \in y \nLeftarrow y \in z_{0}\right) \vee \\
&\left.\left(-x \in y \nLeftarrow-y \in z_{0}\right)\right] .
\end{aligned}
$$

But if $x \in A_{1}^{u}$, then $-x \in A_{1}^{u}$, so (10) implies also

$$
\begin{aligned}
\left(\forall x \in A_{1}^{u}\right)\left(\forall y \in A_{2}^{u}\right)\left[\left\{\emptyset, A_{0}\right\} \subseteq y\right. & \Rightarrow \\
& \left(-x \in y \nLeftarrow y \in z_{0}\right) \vee \\
& \left.\left(x \in y \nLeftarrow-y \in z_{0}\right)\right] .
\end{aligned}
$$

Taking the conjunction of (10) and (11) and applying some logic calculus we get the following:

$$
\begin{gathered}
\left(\forall x \in A_{1}^{u}\right)\left(\forall y \in A_{2}^{u}\right)\left[\left\{\emptyset, A_{0}\right\} \subseteq y \Rightarrow\right. \\
\left(x \in y \Leftrightarrow y \notin z_{0}\right) \wedge\left(-x \in y \Leftrightarrow y \notin z_{0}\right) \vee \\
\left(x \in y \Leftrightarrow y \notin z_{0}\right) \wedge\left(x \in y \Leftrightarrow-y \notin z_{0}\right) \vee \\
\left(-x \in y \Leftrightarrow-y \notin z_{0}\right) \wedge\left(-x \in y \Leftrightarrow y \notin z_{0}\right) \vee \\
\left.\left(-x \in y \Leftrightarrow-y \notin z_{0}\right) \wedge\left(x \in y \Leftrightarrow-y \notin z_{0}\right)\right] .
\end{gathered}
$$

By logic again (12) is written

$$
\begin{aligned}
&\left(\forall x \in A_{1}^{u}\right)\left(\forall y \in A_{2}^{u}\right)\left[\left\{\emptyset, A_{0}\right\} \subseteq y \Rightarrow\right. \\
&\left(x \in y \Leftrightarrow-x \in y \Leftrightarrow y \notin z_{0}\right) \vee \\
&\left(y \notin z_{0} \Leftrightarrow x \in y \Leftrightarrow-y \notin z_{0}\right) \vee \\
&\left(-y \notin z_{0} \Leftrightarrow-x \in y \Leftrightarrow y \notin z_{0}\right) \vee \\
&\left.\left(-x \in y \Leftrightarrow-y \notin z_{0} \Leftrightarrow x \in y\right)\right] .
\end{aligned}
$$

Now each of the first and fourth disjuncts of (13) implies $(x \in y \Leftrightarrow-x \in$ $y$ ), while each of the second and third implies $\left(y \in z_{0} \Leftrightarrow-y \in z_{0}\right)$. So (13) yields

$$
\begin{gathered}
\left(\forall x \in A_{1}^{u}\right)\left(\forall y \in A_{2}^{u}\right)\left[\left\{\emptyset, A_{0}\right\} \subseteq y \Rightarrow\right. \\
(x \in y \Leftrightarrow-x \in y) \vee
\end{gathered}
$$




$$
\left.\left(y \in z_{0} \Leftrightarrow-y \in z_{0}\right)\right]
$$

(14) is equivalently written as follows:

$$
\begin{gathered}
\left(\forall y \in A_{2}^{u}\right)\left[\left\{\emptyset, A_{0}\right\} \subseteq y \Rightarrow\left(\forall x \in A_{1}^{u}\right)(x \in y \Leftrightarrow-x \in y) \vee\right. \\
\left.\left(y \in z_{0} \Leftrightarrow-y \in z_{0}\right)\right] .
\end{gathered}
$$

In order to simplify (15), we give a definition. Given sets $z_{1}, z_{2} \in A_{3}$, let us call $z_{1}$ closed with respect to $z_{2}$, if

$$
\forall y \in z_{2}\left(y \in z_{1} \Longleftrightarrow-y \in z_{1}\right) \text {. }
$$

Let us denote this property by $C\left(z_{1} ; z_{2}\right)$. Obviously $C\left(z_{1}, z_{2}\right)$ is a formula of $L_{\mathrm{TST}}$. Similarly is defined the property $C\left(y_{1} ; y_{2}\right)$ for $y_{1}, y_{2} \in A_{2}$. Note that the subformula $\left(\forall x \in A_{1}^{u}\right)(x \in y \Leftrightarrow-x \in y)$ of (15) says that $y$ is closed w.r.t. $A_{1}^{u}$, i.e., $C\left(y ; A_{1}^{u}\right)$. So $(15)$ is written as follows:

$$
\left(\forall y \in A_{2}^{u}\right)\left[\left\{\emptyset, A_{0}\right\} \subseteq y \wedge \neg C\left(y ; A_{1}^{u}\right) \Rightarrow\left(y \in z_{0} \Leftrightarrow-y \in z_{0}\right)\right] .
$$

Let

$$
X=\left\{y \in A_{2}^{u}:\left\{\emptyset, A_{0}\right\} \subseteq y \wedge \neg C\left(y ; A_{1}^{u}\right)\right\} .
$$

Clearly $X$ is definable in $\mathcal{A}$ and $X \in A_{3}$. Moreover (16) is written

$$
\left.(\forall y \in X)\left(y \in z_{0} \Leftrightarrow-y \in z_{0}\right)\right]
$$

or, equivalently, " $z_{0}$ is closed with respect to $X$ ", i.e.

$$
C\left(z_{0} ; X\right)
$$

Now since $(8) \Rightarrow(18)$, if $z_{0}$ is not closed with respect to $X$, i.e., if $\neg C\left(z_{0} ; X\right)$, then $(8)$ is false, and hence $o^{\mathcal{A}}$ is extendible at $z_{0}$. So it remains to show extendibility when $C\left(z_{0} ; X\right)$ is true.

Suppose $C\left(z_{0} ; X\right)$. We have to show that

$$
\begin{aligned}
&\left(\exists x \in A_{1}^{u}\right)\left(\exists y \in A_{2}^{u}\right)\left[\left\{\emptyset, A_{0}\right\}\right. \subseteq y \\
&\left(x \in y \Leftrightarrow y \in z_{0}\right) \wedge \\
&\left.\left(-x \in y \Leftrightarrow-y \in z_{0}\right)\right] .
\end{aligned}
$$


In view of the definition of $X$ it suffices to show that

$$
(\exists y \in X)\left(\exists x \in A_{1}^{u}\right)\left(y \in z_{0} \wedge x \in y \wedge-x \in y\right)
$$

or

$$
(\exists y \in X)\left(\exists x \in A_{1}^{u}\right)\left(y \notin z_{0} \wedge x \notin y \wedge-x \notin y\right) .
$$

Indeed if $x_{0}, y_{0}$ satisfy (20), then $-y_{0} \in z_{0}$ holds also because $z_{0}$ is closed with respect to $X$, hence

$$
\left(x_{0} \in y_{0} \Leftrightarrow y_{0} \in z_{0}\right) \wedge\left(-x_{0} \in y_{0} \Leftrightarrow-y_{0} \in z_{0}\right)
$$

is true. Moreover $\left\{\emptyset, A_{0}\right\} \subseteq y_{0}$, because $y_{0} \in X$. Therefore $x_{0}, y_{0}$ satisfy (19). Similarly if $x_{0}, y_{0}$ satisfy (21), then by closeness again $-y_{0} \notin z_{0}$, hence

$$
\left(x_{0} \in y_{0} \Leftrightarrow y_{0} \in z_{0}\right) \wedge\left(-x_{0} \in y_{0} \Leftrightarrow-y_{0} \in z_{0}\right)
$$

is true. Therefore (19) holds again.

Claim. $(20) \vee(21)$ is true.

Proof. It suffices to show that the negation of $(20) \vee(21)$ leads to a contradiction. The negation of $(20) \vee(21)$ is the conjunction of

$$
(\forall y \in X)\left(\forall x \in A_{1}^{u}\right)\left(y \notin z_{0} \vee x \notin y \vee-x \notin y\right)
$$

and

$$
(\forall y \in X)\left(\forall x \in A_{1}^{u}\right)\left(y \in z_{0} \vee x \in y \vee-x \in y\right) .
$$

We can rewrite (22) and (23) as follows:

$$
\left(\forall y \in X \cap z_{0}\right)\left(\forall x \in A_{1}^{u}\right)(x \in y \Rightarrow-x \notin y)
$$

and

$$
\left(\forall y \in X \cap-z_{0}\right)\left(\forall x \in A_{1}^{u}\right)(x \notin y \Rightarrow-x \in y) .
$$

(24) and (25) say that $z_{0}$ partitions $X$ into a part $X \cap z_{0}$ consisting of "consistent" $y$ only (i.e., not containing both $x$ and $-x$ for any $x$ ), and a part $X \cap-z_{0}$ consisting of "complete" $y$ only, (i.e., containing at least one of the $x$ and $-x$ for every $x$ ). But this is false, since $X$ must contain also $y$ which are neither consistent nor complete. To show this, we make use of the assumption that $\mathcal{A}$ is the level collapse of an elementary submodel of a full model. Actually this is the only point in the proof where we use this strong hypothesis. (For 
all other purposes, regularity and richness suffice.) Indeed let $\mathcal{A}=l c(\mathcal{B})$, for a countable $\mathcal{B} \preceq\langle\langle D\rangle\rangle$. In $\langle\langle D\rangle\rangle$, the corresponding set $X$ contains sets that are neither consistent nor complete. Namely in $\langle\langle D\rangle\rangle$, using possibly the choice axiom of the ground model, we can find a $y^{*} \in \mathcal{P}^{2}(D)$ such that (a) $\{\emptyset, D\} \subseteq y^{*}$, (b) $\left|y^{*}\right|=\left|-y^{*}\right|$, (c) $\exists x_{1}\left(x_{1} \in y^{*} \wedge-x_{1} \notin y^{*}\right)$ (non-closed), (d) $\exists x_{2}\left(x_{2} \in y^{*} \wedge-x_{2} \in y^{*}\right)$ (non-consistent) and (e) $\exists x_{3}\left(x_{3} \notin y^{*} \wedge-x_{3} \notin y^{*}\right)$ (non-complete). Since $\mathcal{A}=l c(\mathcal{B})$ and $\mathcal{B}$ is countable, by lemma 1.2 there is a $y$ in $\mathcal{A}$ with properties (a)-(e) above. Therefore (24) and (25) lead to a contradiction. This contradiction shows that $(22) \wedge(23)$ is false, and hence $(20) \vee(21)$ is true. This completes the proof of the Claim.

Thus $o^{\mathcal{A}}$ extends at $z_{0}$ in all cases, and this completes the proof of the lemma.

By lemma 3.3, the level collapse of an elementary submodel of a full model is rich. So from lemmas 3.4 and 3.5 we derive immediately the following:

Theorem 3.6 Let $\langle\langle D\rangle\rangle$ be a full model of TST (with infinite D) and let $\mathcal{A}$ be the level collapse of a countable elementary submodel of $\langle\langle D\rangle\rangle$. Then the pair $o^{\mathcal{A}}$ is extendible in $\mathcal{A}$.

\section{2-Extendibility.}

What about 2- (or higher) extendible coherent pairs? Again the simplest question is: Is there a model $\mathcal{A}$ of TST such that $o^{\mathcal{A}}$ is 2-extendible?

2 -extendibility (as well as $n$-extendibility in general) splits again to $A_{i}$-2extendibility, for $i=1,2,3$. Given $\mathcal{A}, o^{\mathcal{A}}$ is $A_{1}$-2-extendible if

$$
\left(\forall x \in A_{1}\right)(\exists q)\left(q \leq o^{\mathcal{A}} \wedge x \in \operatorname{dom}\left(q_{1}\right) \wedge q \text { is extendible }\right) .
$$

The last formula when decompressed becomes:

$\psi_{1}: \quad\left\{\forall x \in A_{1} \exists y \in A_{2} \exists z \in A_{3}\right.$ such that, if $v_{1}=\left\{\emptyset, A_{0}, x,-x\right\}$, $v_{2}=\left\{\emptyset, A_{1}, y,-y\right\}, v_{3}=\left\{\emptyset, A_{2}, z,-z\right\}$, then the pair $v_{1} \stackrel{q_{1}}{\longrightarrow} v_{2} \stackrel{q_{2}}{\longrightarrow} v_{3}$, where $q_{1}$ and $q_{2}$ are the mappings which preserve the orderings of $v_{1}, v_{2}, v_{3}$ as exhibited above, is not only a coherent pair but also an extendible one.

For $A_{2}$-2- and $A_{3}$-2-extendibility of $o^{\mathcal{A}}$ the formulation is similar, except of the string of initial quantifiers. That is, $A_{2}$-2-extendibility is written: 
$\psi_{2}: \quad\left\{\forall y \in A_{2} \exists x \in A_{1} \exists z \in A_{3}\right.$ such that, if $v_{1}=\left\{\emptyset, A_{0}, x,-x\right\}, v_{2}=$ $\left\{\emptyset, A_{1}, y,-y\right\}, v_{3}=\left\{\emptyset, A_{2}, z,-z\right\}$, then $v_{1} \stackrel{q_{1}}{\longrightarrow} v_{2} \stackrel{q_{2}}{\longrightarrow} v_{3}$ is an extendible pair. $\}$

And $A_{3}$-2-extendibility is written:

$\psi_{3}: \quad\left\{\forall z \in A_{3} \exists x \in A_{1} \exists y \in A_{2}\right.$ such that, if $v_{1}=\left\{\emptyset, A_{0}, x,-x\right\}, v_{2}=$ $\left\{\emptyset, A_{1}, y,-y\right\}, v_{3}=\left\{\emptyset, A_{2}, z,-z\right\}$, then $v_{1} \stackrel{q_{1}}{\longrightarrow} v_{2} \stackrel{q_{2}}{\longrightarrow} v_{3}$ is an extendible pair. $\}$

Thus 2-extendibility of $o^{\mathcal{A}}$ amounts to the truth of the formula $\psi_{1} \wedge \psi_{2} \wedge \psi_{3}$. At first sight it looks overwhelmingly involved, but if we find conditions deciding when an arbitrary pair $v_{1} \stackrel{q_{1}}{\longrightarrow} v_{2} \stackrel{q_{2}}{\longrightarrow} v_{3}$ is extendible, things may become easier.

\section{References}

[1] T.E. Forster, Set theory with a universal set, Oxford Logic Guides Vol. 20, Oxford U.P. 1992.

[2] V.N. Grishin, Consistency of a fragment of Quine's NF system, Soviet Mathematics Doklady 10, No 6 (1969), 1387-1390.

[3] V.N. Grishin, The method of stratification in set theory (in Russian), Ph.D. Thesis, Moscow University 1972.

[4] E. Specker, Typical ambiguity, Logic Methodology and Philosophy of Science, Proceedings of the International Congress, Stanford, 1960, Stanford University Press 1962, pp. 116-124. 